\title{
Lúpus eritematoso sistêmico diagnosticado durante a gestação: relato de caso
}

\author{
Systemic lupus erythematosus diagnosed during pregnancy: a case report
}

\author{
Luilson Geraldo Coelho Junior ${ }^{1}$, Gabrielly Borges Machado², \\ Estevão Tavares de Figueiredo ${ }^{3}$, Talitha Araújo Faria ${ }^{4}$
}

\begin{abstract}
Coelho Junior LG, Machado GB, Figueiredo ET, Faria TA. Lúpus eritematoso sistêmico diagnosticado durante a gestação: relato de caso / Systemic lupus erythematosus diagnosed during pregnancy: a case report. Rev Med (São Paulo). 2015 out.-dez.;94(4):289-93.
\end{abstract}

RESUMO: O lúpus eritematoso sistêmico (LES) é uma doença inflamatória crônica de etiologia autoimune, distribuição multissistêmica, cujas manifestações clínicas são bastante variáveis. Fatores genéticos, hormonais e ambientais estão correlacionados com o desenvolvimento da doença, a qual é caracterizada por períodos de remissão e exacerbação. No LES, os fenômenos imunológicos levam à produção de auto-anticorpos, os quais são dirigidos a antígenos nucleares do próprio organismo, resultando em diferentes manifestações clínicas, podendo acometer ossos, articulações, pulmões, fígado, rins e sistema nervoso central. Mulheres em período fértil têm alta prevalência, sendo que, na maior parte dos casos, não têm sua fertilidade comprometida. $\mathrm{O}$ início do quadro durante a gravidez é raro e indica pior prognóstico para mãe e feto. A gravidez está desaconselhada quando a doença está ativa durante a concepção, e quando se associa à nefrite lúpica. Mulheres portadoras de lúpus cursam com gestação de alto risco, haja vista, as gestantes lúpicas apresentarem maior taxa de abortamento espontâneo, restrição de crescimento intra-uterino e fetal, eclâmpsia, morte fetal, parto pré-termo. Há controvérsias sobre o efeito da gestação na atividade da doença, uma vez que, o LES pode afetar a gravidez, e o inverso também pode ocorrer; todavia, o LES não deve ser entendido como uma limitação à gestação, e o acompanhamento da mesma deve ser feito por equipe multiprofissional, assegurando uma gravidez segura para mãe e feto. Apresentamos o caso de uma paciente diagnosticada com LES no primeiro trimestre de gestação e que apresentou evolução clínica e laboratorial rápida e desfavorável, evoluindo com quadro de nefrite lúpica e abortamento.

Descritores: Lúpus eritematoso sistêmico; Gravidez; Cuidado pré-natal; Nefrite lúpica; Pré-eclâmpsia; Eclâmpsia.
ABSTRACT: Systemic lupus erythematosus (SLE) is a chronic inflammatory disease of autoimmune etiology, multisystem distribution, whose clinical manifestations are quite variable. Genetic and environmental factors are correlated with the development of the disease, which is characterized by periods of remission and exacerbation. In SLE, immunological phenomena lead to the production of auto-antibodies, which are directed to nuclear antigens of the organism itself, resulting in different clinical manifestations and may involve the bones, joints, lungs, liver, kidneys and central nervous system. Women of childbearing age have high prevalence, and, in most cases, do not have a compromised fertility. The onset during pregnancy is rare and indicates poor prognosis for mother and fetus. Pregnancy is not recommended when the disease is active at conception. Women with lupus have high-risk pregnancy, because pregnant women lupus has high rates of abortion, intrauterine and fetal growth restriction, preeclampsia, fetal death, preterm delivery. There is controversy about the effect of pregnancy on disease activity, since SLE can affect pregnancy, and the reverse can also occur; however, the SLE should not be understood as a limitation to pregnancy, and the monitoring thereof should be done by multi-professional team, ensuring a safe pregnancy for mother and fetus. We present the case of a patient diagnosed with SLE in the first trimester of pregnancy and that presented clinical and laboratory evolution rapid and unfavourable, evolving with lupus nephritis and abortion.

Keywords: Systemic lupus erythematosus; Pregnancy; Prenatal care; Lupus nephritis; Pre-eclampsia; Eclampsia.

1. Acadêmico de Medicina da Faculdade Atenas, Paractu, MG.

2. Médica Infectologista do Departamento de Infectologia da Faculdade Atenas, Paracatu, MG. E-mail: gabyborges100@hotmail.com.

3. Médico Cardiologista do Departamento de Cardiologia da Faculdade Atenas, Paracatu, MG. E-mail: estevaotavares@cardiol.br.

4. Disciplina de Pensamento Científico da Faculdade Atenas, Paracatu, MG. E-mail: talithabio@yahoo.com.br.

Autor para correspondência: Luilson Geraldo Coelho Junior. Rua Bernardo Caparucho, 30, apartamento 04 - Bela Vista. CEP: 38600000 - Paracatu, MG. E-mail: luilson-junior@hotmail.com. 


\section{INTRODUÇÃO} Lúpus Eritematoso Sistêmico (LES) é uma
doença inflamatória crônica e multissistêmica que acomete principalmente mulheres jovens, em idade reprodutiva. A doença apresenta evolução crônica e caracteriza-se por períodos de atividade e remissão ${ }^{1}$.

O LES é uma doença rara, incidindo, mais freqüentemente, em mulheres na menacme, na proporção de nove a dez mulheres para um homem, e com prevalência variando de 14 a 50/100.000 habitantes, em estudos norteamericanos $^{2}$.

A base fisiopatológica do LES está centrada na autoimunidade, sendo que a hiperreatividade de linfócitos B leva a produção de autoanticorpos e formação de imunocomplexos antígeno anticorpo ${ }^{3}$.

A perda da tolerância imunológica desencadeia a síntese de anticorpos, os quais são direcionados à proteínas do próprio organismo, podendo acometer múltiplos órgãos e sistemas, incluindo ossos, articulações, pulmões, pleura, pericárdio, fígado, rins, sistema nervoso central, pele, trato gastrointestinal ${ }^{4}$.

A gravidez é uma condição fisiológica e compreende um estado de imunomodulação, no qual há alterações neuroimunoendócrinas que levam à supressão de resposta imune do tipo Th1, e prevalência de resposta $T h 2^{5}$. As alterações imunológicas favorecem e asseguram o desenvolvimento da gestação, pois há tolerância aos antígenos paternos e fetais. A intensidade e a qualidade da resposta do sistema imune materno são fundamentais para determinar o sucesso ou não da gravidez ${ }^{6,7}$.

A incidência dessa enfermidade entre as gestantes varia de 1:660 a 1:2.952 ${ }^{8}$. O LES não altera a fertilidade da mulher, porém há um maior risco de consequências materno fetais, decorrentes da atividade da doença durante a gestação, da terapêutica instalada e resposta imunológica desencadeada pela mãe durante a gravidez .

As gestações em mulheres lúpicas são consideradas de alto risco, requerem acompanhamento por equipes multidisciplinares e avaliação durante toda a gestação ${ }^{10}$. No passado as mulheres lúpicas eram orientadas a não engravidar, atualmente, devido maior acurácia diagnóstica, maiores conhecimentos sobre a doença, melhor manejo dos doentes, monitorização e melhores recursos terapêuticos permitiram um prognóstico mais favorável a essas doentes $^{11,12}$.

A agudização da doença no período gestacional implica em pior prognóstico materno-fetal, levando a abortamento espontâneo, restrição de crescimento fetal e uterino, hipertensão gestacional, nefrite lúpica, parto prematuro, lúpus neonatal, mortalidade materna ${ }^{13}$.

Neste relato de caso, o LES, diagnosticado durante a gestação, agravou a função renal, o que levou a instabilidade hemodinâmica. Além disso, esta paciente apresentou uma gestação anterior sem intercorrências, já nessa gestação, apresentou uma evolução clínica e laboratorial rápida e desfavorável, pois a gravidez agravou o LES, o que culminou em abortamento, hemorragia alveolar e nefrite.

\section{RELATO DE CASO}

Paciente do sexo feminino, 28 anos de idade, casada, negra, mãe de um filho de 03 anos, natural e procedente de Paracatu, MG.

Paciente secundigesta, primípara, IG 17 semanas e 6 dias, durante consulta de pré-natal, realizada em unidade básica de saúde (UBS), no mês de maio, apresentava lesões circulares com halo hiperemiado em palmas das mãos e plantas dos pés, além de edema e artralgia em articulações de mãos.A hipótese diagnóstica foi de LES; à pesquisa de autoanticorpos: Anti-SM reagente, títulos maiores que 480 U/mL; FAN- Fator Antinuclear reagente (núcleo e citoplasma reagente), título 1/60, Anti DNA positivo.

Iniciou-se Prednisona 20mg/d. Ao exame clínico: Pressão arterial: 130x90 mmHg, FC:77bpm, FR: 18irpm, Hemograma: Hemácias: 3,35 milhões $/ \mathrm{mm}^{3}$, Hb: 6,70 g/dL, $\mathrm{Hb}: 20,60 \%$; Leucócitos: $5900 \mathrm{~mm}^{3}$, Linfócitos:900 $\mathrm{mm}^{3}$; Plaquetas $169000 \mathrm{~mm}$; Creatinina: 1,4 mg/dL; Ureia: $44 \mathrm{mg} / \mathrm{dL}$.

Nega doenças de base e alergias medicamentosas. Relata gestação anterior sem intercorrências.Nega história familiar de Lúpus Eritematoso Sistêmico (LES). Não faz uso de medicações de uso contínuo como anticoncepcional, aspirina, heparina ou corticóides.

Após uma semana, paciente realizou consulta médica em UBS e foi encaminhada ao Hospital municipal devido intensa anemia, $\mathrm{Hb}: 5 \mathrm{~g} / \mathrm{dl}, \mathrm{Ht}: 15 \%$, além de lombalgia, astenia, dispnéia e oligúria. Recebeu transfusão de concentrado de hemácias, três bolsas, havendo piora da dispnéia e astenia. Paciente não fez uso de glicocorticóide endovenoso e não apresentava sinais de hemólise. Plaquetas: $160000 \mathrm{~mm}^{3}$. Realizado Raio X de Tórax, sem alterações em campos pulmonares.Ausência de hemorragia alveolar.PA: 150x100mmHg e foi iniciado Nifedipino 40mg/d. O diagnóstico estabelecido foi de LES.

Após transfusão sanguínea, o hemograma revelou $\mathrm{Hb}: 6,8 \mathrm{~g} / \mathrm{dl}$, Ht: 18\%, Leucócitos: $12000 \mathrm{~mm}^{3}$, Linfócitos $1484 \mathrm{~mm}^{3}$, Plaquetas: $180000 \mathrm{~mm}^{3}$; Ur: $70 \mathrm{mg} / \mathrm{dl}, \mathrm{Cr}: 3,2 \mathrm{mg} /$ dl, TGO: 29 U/L, TGP 17 U/L, EAS: 20leuc/campo, 10 hem/campo, proteína ++ . Recebeu nova transfusão de concentrado de hemácias, duas bolsas, com piora importante da dispnéia e iniciou com estertores em base pulmonar bilateralmente, Raio X de Tórax sem alterações. Sinais Vitais: PA: 130x80mmHg; FC: 144bpm;FR: 34 irpm. Paciente não fez uso de glicocorticóide venoso, ou outras medicações, pois foi encaminhada para UTI. Paciente não apresentou hemorragia alveolar.

Paciente foi admita em Unidade de Terapia Intensiva 
Coelho Junior LG, et al. Lúpus eritematoso sistêmico diagnosticado durante a gestação.

devido piora hemodinâmica e anúria. Valores de uréia: 82 $\mathrm{mg} / \mathrm{dl}$ e Cr: 3,72 mg/dl, paciente em acidose metabólica com compensação respiratória, mantendo taquidispnéia leve. GASOMETRIA: ph: 7,47, $\mathrm{HCO}^{3}: 10,2, \mathrm{CO}^{2}$ : 14, BE: -12,3. Em acompanhamento conjunto com Ginecologia e mantido suporte intensivo.

Primeiro dia de internação em UTI apresentavase taquidispnéica com importante esforço respiratório, estertores bibasais, em uso de máscara de Venturi a 50\%, e descorada $++/ 4$. Resultados de exame com Uréia: 105 $\mathrm{mg} / \mathrm{dl}, \mathrm{Cr}: 4,01 \mathrm{mg} / \mathrm{dL}$, hipocomplementemia: C3: 44,1 mg/ $\mathrm{dL}, \mathrm{C} 4: 3,8 \mathrm{mg} / \mathrm{dL}$, Proteinúria de 24horas: $320 \mathrm{mg} / 24 \mathrm{hs}$, Anti DNA reagente. Foi iniciada a pulsoterapia com metilpredinisolona $1 \mathrm{~g}$ por dia durante três dias. Discutido com a nefrologia que indicou hemodiálise diária. À avaliação ginecológica BCF: 168 bpm. Mantido suporte intensivo, foi discutida prioridade materna com a equipe médica. Estabeleceu o diagnóstico de nefrite lúpica.

No segundo dia de UTI refere melhora da dispneia. Avaliação ginecológica apresentando $\mathrm{BCF}=160 \mathrm{bpm}$. Orientado ajuste de metildopa 6/6 horas e nifedipino 20 mg 8/8 horas, mantido pulsoterapia.

No terceiro dia de UTI paciente estava hemodinamicamente estável. Diálise mantida. BCF: 142 bpm. Mantendo bom padrão respiratório. Último dia de pulsoterapia.

Paciente estável hemodinamicamente, hipocorada $(++/++++)$, discretamente dispneica.Queixa-se de dor em mãos. Ausência de edema em MMII. Realizou HD com UF: $1000 \mathrm{ml}$. Apresentou melhora do débito urinário com diurese de $500 \mathrm{ml} / 12 \mathrm{~h}$. Iniciado a corticoterapia de manutenção com Prednisona $60 \mathrm{mg} /$ dia oral.

No quarto dia, paciente estável hemodinamicamente, com dispneia moderada, em uso de máscara de venturi 50\%, com edema moderado de face. Detectou-se proteinúria $24 \mathrm{~h}$ : $535,5 \mathrm{mg} / 24 \mathrm{~h}$.

No quinto dia, paciente estava muito ansiosa e queixou-se de mal estar. Apresentando taquipnéia, esforço intenso, com utilização de musculatura acessória, dependente de $\mathrm{O} 2$ por venturi 50\%, com SatO2: 92\%. Avaliação pela Ginecologia identifica sinais de sofrimento fetal. Avalia-se a necessidade de IOT/VM. Equipe multidisciplinar discute o caso, considerado de alto risco para mãe e feto, e decidem por priorizar a vida materna.

No sexto dia, paciente queixa-se de mal estar súbito, caracterizado por calor intenso, cólicas abdominais e sudorese profusa. Apresentou PA: 200/120 mmHg. Foi realizado bolus de Hidralazina. Paciente evoluiu com piora do padrão respiratório com necessidade de IOT. Ao realizar o procedimento paciente apresentou quadro de hemorragia alveolar profusa. Apresentou PCR por hipóxia, a qual foi revertida após 2 minutos de manobras de RCP. Paciente em estado gravíssimo, sob efeito de sedação com Fentanil $5 \mathrm{ml} / \mathrm{h}$ e Midazolan 20ml/h.

No sétimo dia de internação paciente apresentando instabilidade hemodinâmica com necessidade de droga vasoativa (Noradrenalina) e melhora do sangramento pulmonar. Mantido suporte intensivo, hemodiálise diária e acompanhamento multiprofissional. Paciente apresentou abortamento espontâneo, e está hemodinamicamente estável.

Após o aborto, a paciente apresentou rápida melhora do estado hemodinâmico e do quadro renal, não apresentou hipertensão arterial nem atividade lúpica.

\section{DISCUSSÃO}

O LES por si só não constitui contra-indicação à gravidez, exceto quando a paciente apresenta: hipertensão pulmonar e doença pulmonar restritiva severa, insuficiência cardíaca, insuficiência renal crônica, pré eclampsia grave ou Síndrome caracterizada por Hemólise, níveis elevados de enzimas hepáticas e baixos níveis de plaquetas (Hellp), prévios apesar de terapêutica com aspirina e heparina, acidente vascular cerebral nos últimos 6 meses, agudização severa da doença nos últimos 6 meses $^{10}$.

A gravidez em mulheres lúpicas apresenta duas a quatro vezes mais complicações obstétricas do que em gestantes não lúpicas, associa-se a um aumento do risco de cerca de 20 vezes na mortalidade materna ${ }^{15,21}$.

É recomendado adiar a gravidez até o LES estar numa fase quiescente, planejar a gestação, realizar contracepção eficaz, o pré-natal deve ser acompanhado por equipe multidisciplinar, além de orientar a mãe sobre os riscos da gestação em relação à ativação da doença ${ }^{14}$. O planejamento adequado da gravidez é fundamental, pois está relacionado com o sucesso da gestação, redução de possíveis danos materno-fetais ${ }^{13}$. No caso descrito, a paciente desconhecia ser portadora da doença, sendo que esta se manifestou, somente, na segunda gestação.

Embora haja melhora no prognóstico de mulheres lúpicas durante a gestação, estas continuam a apresentar famílias pequenas e ao comparar o número de nascimentos com a população geral, mulheres lúpicas possuem menor número de nascimentos ${ }^{16}$.

O LES é uma doença auto-imune, com apresentação clínica e patológica variável; o acometimento renal é comum, variando de $30 \%$ a $90 \%$ em pacientes com $\mathrm{LES}^{3,17}$. Assim sendo, constitui um fator de agravo quanto a morbimortalidade, haja vista, sobrepor a hipertensão arterial, síndrome nefrótica e insuficiência renal ${ }^{13,17,18,19}$.

O tratamento da nefropatia visa controlar o processo inflamatório e evitar a evolução para a insuficiência renal crônica. A gestação em pacientes com nefrite lúpica está associada a elevado risco materno-fetal e piora das manifestações renais, sendo a gestação de alto risco $^{12,13,18}$. Neste trabalho, a nefrite lúpica representou um pior prognóstico durante a gestação, devido a piora hemodinâmica da mãe.

A paciente apresentou a síndrome pulmão - rim, a qual 
Coelho Junior LG, et al. Lúpus eritematoso sistêmico diagnosticado durante a gestação.

é caracterizada por hemorragia alveolar e glomerulonefrite; esta síndrome apresenta alta morbimortalidade, e a microangiopatia renal e pulmonar, associada a depósito de imunocomplexos, levam a ativação do sistema imunológico, levando a insuficiência respiratória e renal ${ }^{20}$.

Há uma controvérsia sobre o efeito da gestação na atividade da doença, pois o LES pode afetar a gravidez e o inverso pode ocorrer, porém a concepção não necessariamente agrava a doença. Índices maiores que $80 \%$ de êxito na gestação de pacientes lúpicas, portanto o LES não deve ser entendido como limitação à gestação $0^{1,21}$.

Há autores que defendem o aumento da atividade lúpica durante a gestação, já outros não evidenciaram diferenças em relação à freqüência de crises da doença em pacientes grávidas e não-grávidas ${ }^{19,22}$. No caso descrito, a paciente foi diagnosticada com LES durante consulta de pré-natal apresentando artralgia, lesões cutâneas e evoluiu com nefrite lúpica, evidenciando uma evolução de sinais e sintomas rápida.

Os surtos da doença são na maioria de intensidade leve a moderada e acometem 15 a $74 \%$ das gestantes lúpicas. ${ }^{23}$ As taxas de exacerbação do LES estão correlacionadas com a atividade da doença no momento da concepção, sendo mais freqüentes em pacientes com algum acometimento renal, como por exemplo, nefrite lúpica, doenças glomerulares, hipertensão arterial sistêmica moderada a grave? .

Ao correlacionar o momento da concepção e atividade da doença, 58\% das mulheres que apresentam atividade lúpica antes da concepção, enfrentarão mais atividade da doença na gravidez, enquanto $8 \%$ das mulheres com lúpus inativo na época da concepção, enfrentarão menos reativação. ${ }^{15}$

O abortamento espontâneo constitui uma complicação decorrente da ativação da doença no período gestacional. A presença de hipocomplementemia de C3, a nefrite lúpica materna, hipertensão gestacional corroboram para este desfecho ${ }^{17}$.

A pré-eclampsia é caracterizada por aumento dos níveis pressóricos, edema e proteinúria (superior a $300 \mathrm{mg} / 24$ horas); na eclâmpsia a paciente apresenta convulsões, que geralmente, são precedidas de cefaleia, dor epigástrica, hiperreflexia e hemoconcentração, podendo ocorrer subitamente em pacientes assintomáticas. ${ }^{13} \mathrm{O}$ acometimento no sistema nervoso central de pacientes lúpicos é caracterizado por convulsões, cefaleia e infarto cerebral, estas manifestações quando presentes indicam atividade da doença. Portanto, a eclâmpsia e pré-eclâmpsia são os principais diagnósticos diferenciais ${ }^{24}$.

A síndrome HELLP é definida pela presença de hemólise, elevação de enzimas hepáticas e trombocitopenia em gestante com toxemia. A incidência é de aproximadamente $20 \%$ dos casos de pré- eclampsia grave e está associada à grande morbidade materna e perinatal ${ }^{25}$.

Os principais diagnósticos diferenciais são: esteatose hepática aguda, púrpura trombocitopênica trombótica, síndrome hemolítico urêmica, lúpus eritematoso sistêmico, síndrome antifosfolipídio, púrpura trombocitopênica imune $^{26}$.

É importante conhecer os diagnósticos diferenciais da Síndrome HELLP, pois os sintomas, sinais e achados laboratoriais é comum a diversas doenças.

As gestações em pacientes lúpicas são consideradas de alto risco, por isso o planejamento adequado é fundamental para o seu sucesso. A doença quando ativa contra-indica engravidar. Portanto, o LES não deve ser entendido como limitação à gestação, deve-se controlar a doença e acompanhar rigorosamente a gestante lúpica assegurando uma gestação bem sucedida e um bom desfecho materno-fetal.

Este trabalho evidencia a gravidade da ativação do LES durante a gestação, levando à descompensação renal e hematológica, além de apresentar a síndrome pulmãorim, uma síndrome rara. Este relato contribui para reforçar a importância do pré-natal na saúde materno-fetal. Além disso, se faz necessário o tratamento precoce; pois aumenta a sobrevida, além de melhorar o prognóstico da doença, principalmente nos casos graves de ativação do LES.

Conflito de interesse: Os autores declaram não haver conflito de interesse.

\section{REFERÊNCIAS}

1. Lisboa A, Brito I. Lúpus Eritematoso sistêmico e gravidez: implicações terapêuticas. Arq Med. 2014;28(1):18-24. Disponível em: http://www.scielo.gpeari.mctes.pt/scielo. php?pid=S0871-34132014000100004\&script=sci_arttext.

2. Rus V, Maury EE, Hochberg MC. The epidemiology of systemic lupus erythematosus. In: Wallace DJ, Hahn BH, editors. Dubois lupus erythematosus. 7th ed. Philadelphia: Lippincott Williams \& Wilkins; 2007. p.34-44.

3. Lima GG, Hissa MN, Lima MG, de Figueiredo AAF. Nefropatia lúpica. Pesq Med. 2008;2(3).
4. Borba EF. Consenso de lúpus eritematoso sistêmico. Rev Bras Reumatol. 2008;48(4):196-207. Disponível em: http:// www.scielo.br/pdf/rbr/v48n4/v48n4a02.pdf.

5. Warren JB, Silver RM. Autoimmune disease in pregnancy: systemic lupus erythematosus and antiphospholipid syndrome. Obstet Gynecol Clin N Am. 2004;31:345-72. doi:10.1016/j.ogc.2004.03.007.

6. Baxter AG, Smyth MJ. The role of NK cells in autoimmune disease. Autoimmunity. 2002;35:1-14. doi: 10.1080/08916930290005864. 
Coelho Junior LG, et al. Lúpus eritematoso sistêmico diagnosticado durante a gestação.

7. Pereira AC, Jesus NR, Lage LV, Levy RA. Imunidade na gestação normal e na paciente com lúpus eritematoso sistêmico (LES). Rev Bras Reumatol. 2005;45(3):134-40. http://dx.doi.org/10.1590/S0482-50042005000300008.

8. Rahman FZ, Rahman J, Al-Suleiman SA, Rahman MS. Pregnancy outcome in lupus nephropathy. Arch Gynecol Obstet. 2005;271(3):222-6. doi: 10.1007/s00404-0030574-x.

9. Meyer O. Making pregnancy safer for patients with lupus. Joint Bone Spine. 2004;71(3):178-82. doi:10.1016/S1297319X(03)00155-6.

10. Ruiz-Irastorza G, Khamashtama. Lupus and pregnancy: integrating clues from the bench and bedside. Eur J Clin Invest. 2011;41(6):672-8. doi: 10.1111/j.13652362.2010.02443.x.

11. Gordon PA, Beedham T, Khamashta M, D’Cruz D. Systemic lupus erythematosus in pregnancy. Obstet Gynaecol. 2004;6:80-7. doi: 10.1576/toag.6.2.80.26982.

12. Khamashta MA. Systemic lupus erythematosus and pregnancy. Best Pract Res Clin Rheumatol. 2006;20(4):68594. doi:10.1016/j.berh.2006.04.003.

13. Amadatsu CT, Andrade JQ, Zugaib M. Atividade lúpica durante a gestação. Femina. 2009;37(2):115-9. Disponível em: http://www.febrasgo.org.br/site/wp-content/ uploads/2013/05/Feminav37n2p115-9.pdf.

14. Peart E, Clowse MEB. Systemic lupus erythematosus and pregnancy outcomes: an update and review of the literature. Curr Opin Rheumatol. 2014;26(2). doi: 10.1097/ BOR.0000000000000030.

15. Clowse ME, Jamison M, Myers E, James AH. A national study of the complications of lupus in pregnancy. Am J Obstet Gynecol. 2008;199(2):127.e1-6. doi: 10.1016/j. ajog.2008.03.012.

16. Vinet E, Clarke AE, Gordon C, Urowitz MB, Hanly JG, Pineau CA, Isenberg D, Rahman A, Wallace D, Alarcon Gs, Bruce I, Petri M, Dooley MA, Kalunian K, Maddison P, Aranow C, Van Vollenhoven R, Bernatsky S. Decreased live births in women with systemic lupus erythematosus. Arthritis Care Res. 2011;63(7):1068-72. doi: 10.1002/ acr.20466.

17. Gadelha PS, Costa AG, Moura SRM, Tejo Neto WR.
Complicações e manejo do lúpus eritematoso sistêmico na gestação. Femina. 2008;36(1):55-9. Disponível em: http:// www.febrasgo.org.br/site/wp-content/uploads/2013/05/ Femina-1_janeiro_55-59.pdf.

18. Cruz BA. Atualização em lúpus eritematoso sistêmico, síndrome antifosfolípide e gravidez. Rev Bras Reumatol. 2005;45(3):169-73. http://dx.doi.org/10.1590/S048250042005000300013 .

19. Petri M. Prospective study of systemic lupus erythematosus pregnancies. Lupus. 2004; 13(9):688-9. doi: 10.1191/09612033031u2006oa.

20. Lee JG, Joo KW, Chung WK, Jung YC, Zheung SH, Yoon $\mathrm{HJ}$, et al. Diffuse alveolar hemorrhage in lupus nephritis. Clin Nephrol. 2001;55:282-8.

21. Sociedade Brasileira de Reumatologia. Lúpus eritematoso sistêmico: acometimento cutâneo/articular. Rev Assoc Med Bras. 2006, 52(6): 375-88. http://dx.doi.org/10.1590/ S0104-42302006000600012.

22. Georgiou PE, Politi EN, Katsimbri P, Sakka V, Drosos AA. Outcome of lupus pregnancy: a controlled study. Rheumatology. 2000;39(9):1014-9. doi: 10.1093/ rheumatology/39.9.1014.

23. Chakravarty EF, Colon I, Lengen ES, Nix DA, El-Sayed YY, Genovese MC, et al. Factors that predict prematurity and preeclampsia in pregnancies that are complicated by systemic lupus erythematosus. Am J Obstet Gynecol. 2005;192(6):1897-904. doi:10.1016/j.ajog.2005.02.063.

24. Cruz BA. Atualização em lúpus eritematoso sistêmico, síndrome antifosfolípide e gravidez. Rev Bras Reumatol. 2005;45(3):169-73. http://dx.doi.org/10.1590/S048250042005000300013 .

25. Isler CM, Rinehart BK, Terrone DA, Martin RW, Magann EE, Martin JN, Jr. Maternal mortality associated with HELLP (hemolysis, elevated liver enzymes, and low platelets) syndrome. Am J Obstet Gynecol.1999;181:924-8

26. Sibai B. Diagnosis, controversies, and management of syndrome of hemolysis, elevated liver enzymes, and low platelet count. Obstet Gynecol. 2004;103(5 Pt 1):981-91. doi: 10.1097/01.AOG.0000126245.35811.2a. 